\title{
Characterization of the zinc metalloprotease of Streptococcus suis serotype 2
}

\author{
Audrey Dumesnil ${ }^{1,2}$, Jean-Philippe Auger ${ }^{1,2}$, David Roy $^{1,2}$, Désirée Vötsch $^{3}$, Maren Willenborg $^{3}$, \\ Peter Valentin-Weigand ${ }^{3}$, Pyong Woo Park ${ }^{4}$, Daniel Grenier ${ }^{1,5}$, Nahuel Fittipaldi ${ }^{6}$, Josée Harel ${ }^{1,2}$ \\ and Marcelo Gottschalk ${ }^{1,2^{*}}$
}

\begin{abstract}
Streptococcus suis is a swine pathogen and zoonotic agent responsible for meningitis and septic shock. Although several putative virulence factors have been described, the initial steps of the $S$. suis pathogenesis remain poorly understood. While controversial results have been reported for a S. suis serotype 2 zinc metalloprotease (Zmp) regarding its IgA protease activity, recent phylogenetic analyses suggested that this protein is homologous to the ZmpC of Streptococcus pneumoniae, which is not an IgA protease. Based on the previously described functions of metalloproteases (including IgA protease and $\mathrm{ZmpC}$ ), different experiments were carried out to study the activities of that of $S$. suis serotype 2 . First, results showed that $S$. suis, as well as the recombinant $Z m p$, were unable to cleave human $\lg A_{1}$, confirming lack of IgA protease activity. Similarly, S. suis was unable to cleave P-selectin glycoprotein ligand-1 and to activate matrix metalloprotease 9 , at least under the conditions tested. However, $S$. suis was able to partially cleave mucin 16 and syndecan-1 ectodomains. Experiments carried out with an isogenic $\Delta z m p$ mutant showed that the Zmp protein was partially involved in such activities. The absence of a functional Zmp protein did not affect the ability of S. suis to adhere to porcine bronchial epithelial cells in vitro, or to colonize the upper respiratory tract of pigs in vivo. Taken together, our results show that $\mathrm{S}$. suis serotype $2 \mathrm{Zmp}$ is not a critical virulence factor and highlight the importance of independently confirming results on $\mathrm{S}$. suis virulence by different teams.
\end{abstract}

\section{Introduction}

Streptococcus suis is a swine pathogen responsible for cases of meningitis, arthritis, endocarditis, and sudden death in post-weaned piglets. It is responsible for substantial economic losses to the porcine industry and it also represents a serious problem due to the routine use of antimicrobials in the field in attempts to control the infection [1]. It is also an emerging zoonotic agent causing meningitis and septic shock in individuals associated with the swine/pork industry in Western countries or among the general population in some Asian countries [1]. A total of 35 capsular-based serotypes have been reported, with certain of these having recently been

\footnotetext{
*Correspondence: marcelo.gottschalk@umontreal.ca

1 swine and Poultry Infectious Diseases Research Center (CRIPA), Department of Pathology and Microbiology, Faculty of Veterinary Medicine, University of Montreal, Saint-Hyacinthe, QC, Canada Full list of author information is available at the end of the article
}

described as belonging to other bacterial species [2]. Of the different serotypes, serotype 2 is the most frequently isolated from diseased pigs and humans worldwide [1]. Serotype 2 strains differ greatly in terms of virulence potential and geographic distributions and it can be further classified into different sequence types (ST) based on the multilocus sequence typing (MLST) scheme. Indeed, most virulent strains isolated in Europe and Asia belong to ST1, whereas ST25 and ST28 strains, considered as less virulent, are mainly present in North America [3]. ST7 serotype 2 strains, responsible for at least two major outbreaks of human S. suis infections in China, are considered highly virulent [4].

The initial mechanisms involved in S. suis colonization of the host remain poorly known, with the pathogen being able to survive in the tonsils of swine for long periods of time [5]. S. suis has been described to colonize and interact with epithelial cells and mucus of the host upper respiratory tract in order to reach the bloodstream,

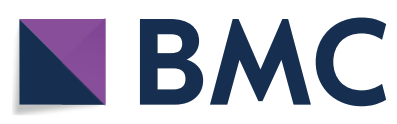

(c) The Author(s) 2018. This article is distributed under the terms of the Creative Commons Attribution 4.0 International License (http://creativecommons.org/licenses/by/4.0/), which permits unrestricted use, distribution, and reproduction in any medium, provided you give appropriate credit to the original author(s) and the source, provide a link to the Creative Commons license, and indicate if changes were made. The Creative Commons Public Domain Dedication waiver (http://creativecommons.org/ publicdomain/zero/1.0/) applies to the data made available in this article, unless otherwise stated. 
where it resists phagocytosis and killing [5]. Replication in blood and systemic dissemination allow $S$. suis to subsequently invade the central nervous system and cause meningitis [6]. Over the years, different bacterial components have been suggested to be involved in the S. suis pathogenesis, including the capsular polysaccharide, the suilysin, the extracellular protein factor (EF), and the muramidase-released protein [7]. However, controversy continues to persist regarding the role of so-called critical S. suis virulence factors [7].

Type A immunoglobulins (IgA) are the predominant immunoglobulin class produced by mucosa-associated lymphoid tissues. They may prevent the adhesion of microorganisms to epithelial cells and consequently facilitate their elimination from the host [5]. In the case of $S$. suis, the secretion of an active human IgA $_{1}$ protease, a zinc-dependent metalloprotease ( $\mathrm{Zmp}$ ) encoded by the iga gene, has been reported in a serotype 2 ST7 strain [8]. The decreased lethality in pigs following intranasal inoculation of an $\Delta i g a$ mutant strain suggested that mucosal IgAs play a crucial role in resistance to $S$. suis invasion and host dissemination $[8,9]$. However, this conclusion may be questionable based on three main considerations: firstly, porcine specific or cross-reactive IgAs against S. suis have never been documented [5]; secondly, no IgA protease activity against human IgAs was detected in any of the $S$. suis strains evaluated in a subsequent study [10], and thirdly, in silico amino acid sequence analysis, as well as structural homology comparisons, do not support the notion that the Zmp encoded by gene iga can have IgA protease activity. In fact, Zmps have been well described in Streptococcus pneumoniae and are classified into four distinct groups: ZmpA (IgA protease), ZmpB, $\mathrm{ZmpC}$, and ZmpD [10]. Similar to S. pneumoniae Zmps, that of $S$. suis is a membrane protein attached by a cellwall LPXTG-anchored motif that possesses G5 tandem repeats and a M26 protease active site. This catalytic site is characterized by a HEMVH motif, which is a key characteristic of the S. pneumoniae ZmpC (but not ZmpA) [8, 10]. In accordance, phylogenetic studies have classified the S. suis Zmp as an homologue of the S. pneumoniae $\mathrm{ZmpC}$ based on genomic sequence similarities [10]. To avoid confusion, we will hereafter refer to the factor as Zmp. Importantly, the gene coding for this protein ( $z m p)$ was found to be present in several of the genomes of 300 S. suis strains recently analyzed [11].

Streptococcus pneumoniae $\mathrm{ZmpC}$ has been described to possesses different activities, which include activation of matrix metalloproteinase 9 (MMP-9) and cleavage of P-selectin glycoprotein ligand-1 (PSGL-1), mucin 16 (MUC16), and syndecan-1 (SDC-1) ectodomains [10, 12-15]. Consequently, given the genetic sequence similarities between Zmps of S. suis and S. pneumoniae, it is possible that this protein could have an important impact on the first steps of S. suis pathogenesis. Moreover, some of these functions could also play important roles in invasion of the central nervous system [16]. However, the actual role of S. suis Zmp in the pathogenesis of the infection has not been completely evaluated. Finally, it is still not clear if Zmp (or S. suis in general) is able to present an IgA protease activity.

In the present study, putative functions as well as the role in virulence of the $S$. suis $\mathrm{Zmp}$ were studied. We report that $S$. suis not only does not cleave human $\operatorname{Ig} \mathrm{A}_{1}$, but it is also unable to cleave PSGL-1 ectodomains or to activate MMP-9. However, $\mathrm{Zmp}$ is responsible, at least in part, for the S. suis cleavage of MUC16 and SDC-1 ectodomains, though this activity does not appear to have a critical impact on $S$. suis serotype 2 colonization of the upper respiratory tract nor virulence.

\section{Materials and methods}

\section{Bacterial strains, plasmids, and growth conditions}

The strains and plasmids used in this study are listed in Table 1 . The virulent $S$. suis serotype 2 strain P1/7 was used for the construction of an isogenic $z m p$-deficient mutant. The S. pneumoniae strain TIGR4 (American Type Culture Collection, Manassas, VA, USA) was used as a positive control for most activation and cleavage assays. Streptococci were grown in Todd-Hewitt broth (THB) until exponential growth phase or agar (THA) for $18 \mathrm{~h}$ (Becton-Dickinson, Sparks, MD, USA) $37^{\circ} \mathrm{C}$ with $5 \% \mathrm{CO}_{2}$, with the exception of the SDC-1 ectodomain cleavage experiment (see below), for which bacteria were used in stationary growth phase as previously described for other pathogens [12, 17, 18]. Escherichia coli strains were grown in Luria-Bertani (LB) broth or agar (Becton-Dickinson) at $37^{\circ} \mathrm{C}$. When needed, antibiotics were added at the following concentrations: for S. suis, spectinomycin at $100 \mu \mathrm{g} / \mathrm{mL}$; for $E$. coli, kanamycin at $25 \mu \mathrm{g} /$ $\mathrm{mL}$ and ampicillin, spectinomycin and carbenicillin at $50 \mu \mathrm{g} / \mathrm{mL}$ (Sigma, Oakville, ON, Canada).

\section{DNA manipulations}

Streptococcus suis genomic DNA was extracted using InstaGene Matrix solution (Biorad Laboratories, Hercules, CA, USA). Plasmid minipreparations were performed with the QIAprep Spin Miniprep Kit (Quiagen, Valencia, CA, USA). Restriction and DNA-modifying enzymes were purchased from Fisher Scientific (Ottawa, ON, Canada) and used according to the manufacturer's recommendations. Oligonucleotide primers (listed in Table 2) were obtained from Integrated DNA Technologies (Coralville, IA, USA). Polymerase chain reactions (PCR) were carried out using the iProof high-fidelity DNA polymerase (BioRad Laboratories, 
Table 1 Bacterial strains and plasmids used in this study

\begin{tabular}{|c|c|c|}
\hline Strains/plasmids & General characteristics & Source/references \\
\hline \multicolumn{3}{|l|}{ Escherichia coli } \\
\hline TOP 10 & $\begin{array}{l}\mathrm{F}^{-} \text {mrcA } \triangle(\mathrm{mrr}-\mathrm{hsdRMS}-\mathrm{mcrBC}) \varphi 80 \text { lacZ } \triangle \mathrm{M} 15 \Delta \mathrm{lacX} 74 \text { recA1 araD139 } \triangle \text { (ara-leu) } 7697 \text { galU galK rpsL } \\
\left(\mathrm{Str}^{\mathrm{R}}\right) \text { endA1 nupG }\end{array}$ & Invitrogen \\
\hline BL21 & $\mathrm{F}^{-}$ompT hsdS $\left(\mathrm{r}_{B}^{-}, \mathrm{m}_{B}^{-}\right)$gal dem rne131 (DE3) & Invitrogen \\
\hline \multicolumn{3}{|l|}{ Streptococcus suis } \\
\hline $\mathrm{P} 1 / 7$ & Wild-type, virulent European serotype 2 ST1 strain isolated from pig with meningitis & [56] \\
\hline$\Delta z m p$ & Isogenic mutant strain derived from P1/7; in frame deletion of zmp gene & This work \\
\hline comp $\triangle z m p$ & Mutant $\triangle z m p$ complemented with $p M X z m p$ complementation vector & This work \\
\hline SC84 & Highly virulent clonal serotype 2 ST7 strain isolated from the 2005 human outbreak in China & [57] \\
\hline \multicolumn{3}{|c|}{ Streptococcus pneumoniae } \\
\hline TIGR4 & Virulent serotype 4 strain isolated from human blood in Norway & ATCC \\
\hline \multicolumn{3}{|l|}{ Plasmids } \\
\hline PCR2.1 & $\mathrm{Ap}^{r}, \mathrm{Km}^{r}$, oriR(f1) MCS oriR (ColE1) & Invitrogen \\
\hline pSET4s & $\begin{array}{l}\text { Thermosensitive vector for allelic replacement in S. suis. Replication functions of pG + host3, MCS oriR } \\
\text { pUC19 lacZ Sp }\end{array}$ & [20] \\
\hline $\mathrm{pMX} 1$ & Replication functions of pSSU1, MCS pUC19 lacZ SpR, malX promoter of S. suis, derivative of pSET2 & {$[20,58]$} \\
\hline pET101 & Apr', pBR322 ori, T7 promotor & Invitrogen \\
\hline $\mathrm{p} 4 \triangle z m p$ & pSET4 s carrying the construct of zmp for allelic replacement & This work \\
\hline pMXzmp & pMX1 complementation vector carrying intact zmp gene & This work \\
\hline pET101zmp & pET101 carrying zmp gene for protein production & This work \\
\hline
\end{tabular}

Table 2 Oligonucleotide primers used in this study

\begin{tabular}{|c|c|c|}
\hline Primer name & Sequence $\left(5^{\prime}-3^{\prime}\right)$ & Construct \\
\hline zmp-ID1 & CCTTGGTTTAGATGCCC & $\mathrm{p} 4 \Delta z m p$ \\
\hline zmp-ID2 & CTGAGACAAGTCCCACT & $\mathrm{p} 4 \Delta z m p$ \\
\hline zmp-ID3 & CAAGCCTTGATGAACTCTAC & $\mathrm{p} 4 \Delta z m p$ \\
\hline zmp-ID4 & GCTATTCCTAGCTTCTACCT & $\mathrm{p} 4 \Delta z m p$ \\
\hline zmp-ID5 & TGTTTCTTTCATCCTTGACAG & $\mathrm{p} 4 \Delta z m p$ \\
\hline zmp-ID6 & CTTGTTAATGATTATTTAATAAGTTGTTACTCCCTAAAATAG & $\mathrm{p} 4 \Delta z \mathrm{mp}$ \\
\hline zmp-ID7 & TAGGGAGTAACAACTTATTAAATAATCATTAACAAGTTGGTC & $\mathrm{p} 4 \Delta z \mathrm{mp}$ \\
\hline zmp-ID8 & ATCTGGCTCATCCATGAC & $\mathrm{p} 4 \Delta z \mathrm{mp}$ \\
\hline pMX1-cpec-F & AAATAGTATAGAAACGGCATGCAAGCTTGG & pMXzmp \\
\hline $\mathrm{pM} \times 1-$ cpec- $\mathrm{R}$ & TCGGAGGTCCTTTAGCCCGGGTACCGAGCT & pMXzmp \\
\hline zmp-cpec-F & CCAAGCTTGCATGCCGTTTCTATACTATTT & pMXzmp \\
\hline zmp-cpec-R & AGCTCGGTACCCGGGCTAAAGGACCTCCGA & pMXzmp \\
\hline pET101-zmp-F & CACCATGGCTCGATATAACCATGCAATC & pET101zmp \\
\hline pET101-zmp-R & TGGGTTAAAAATCGATGTTCTG & pET101zmp \\
\hline
\end{tabular}

Mississauga, ON, Canada) or the Taq DNA polymerase (New England Biolabs, Ipswich, MA, USA). Amplification products were purified using the QIAquick PCR Purification Kit (Qiagen) and sequenced with an ABI 310 automated DNA sequencer and the ABI PRISM dye terminator cycle sequencing kit (Applied Biosystems, Foster City, CA, USA).
Construction of the S. suis zmp-deficient $(\Delta z m p)$ mutant and complemented strain

Precise in-frame deletion of the $z m p$ gene was achieved using splicing-by-overlap-extension PCR [19]. Overlapping PCR-products generated by PCR were cloned into the plasmid pCR2.1 (Invitrogen), extracted using EcoRI, and cloned into the thermosensitive E. coli-S. suis shuttle 
vector pSET4s, as previously described [20]. The resulting mutation vector $\mathrm{p} 4 \Delta z m p$ was electroporated into competent S. suis recipient cells. Allelic replacement and deletion of the gene was confirmed by PCR and sequence analysis.

Circular polymerase extension cloning (CPEC) was used for the construction of the complementation vector of $z m p$ gene, as described previously [21]. Briefly, the complementation vector pMX1, which possesses a malQ inducible promoter, was linearized by PCR and the complete $z m p$ gene amplified. Overhanging primers were used to ligate the gene into the linearized pMX1. The construction was then purified and transformed into electrocompetent $E$. coli MC1061. The complemented $\Delta z m p$ (comp $\Delta z m p$ ) mutant was obtained by transformation into electrocompetent $S$. suis $\Delta z m p$. Obtention of comp $\Delta z m p$ was confirmed by PCR and DNA sequencing analysis.

Absence of Zmp expression in the $\Delta z m p$ mutant and complementation of $z m p$ gene were confirmed by immunoblot as previously described [22], using antisera from rabbits immunized with the recombinant Zmp protein (see below). Expression of a capsular polysaccharide in the S. suis wild-type strain as well as in both the $\Delta z m p$ mutant and comp $\Delta z m p$ complemented strains (already described as a major virulence factor [23]) was confirmed by hydrophobicity and coagglutination assays using serotype 2 antiserum, as previously described [24]. For the hydrophobicity assay, $10 \mathrm{~mL}$ overnight cultures were centrifuged, washed, and resuspended in phosphateurea-magnesium sulfate buffer $\left(96 \mathrm{mM} \mathrm{K}_{2} \mathrm{HPO}_{4}, 53 \mathrm{mM}\right.$ $\mathrm{KH}_{2} \mathrm{PO}_{4}, 30 \mathrm{mM}$ urea, and $0.8 \mathrm{mM} \mathrm{MgSO}$ ) at an optical density (OD) $660 \mathrm{~nm}$ of 0.6. Four hundred microliter of $n$-hexadecane (Sigma) was then add to $3 \mathrm{~mL}$ of the bacterial suspensions, which were vortexed for $2 \mathrm{~min}$, and the OD of the aqueous phase measured. The percentage of hydrophobicity was determined using the following equation: (initial OD - final OD)/initial OD $\times 100 \%$.

\section{Expression and purification of the recombinant Zmp protein}

The region corresponding to the $z m p$ gene, excluding the region encoding the LPVTG motif (to facilitate the purification of the recombinant protein from $E$. coli), was amplified by PCR and directly cloned into the pET101 vector (Invitrogen), which possesses a C-terminal Histag, according to the manufacturer's instructions. Protein production was induced in the E. coli BL21 (DE3) strain using $0.5 \mathrm{mM}$ IPTG for $4 \mathrm{~h}$, after which cells were lysed by sonication. Cell lysates were used to purify the recombinant Zmp protein by His-Bind Resin Chromatography Kit (Novagen, Madison, WI, USA) according to manufacturer's instructions and dialysed. Protein purity was confirmed by SDS-PAGE and Western blot using an anti-His-tag antibody (R\&D Systems, Minneapolis, MN, USA). The purified recombinant protein was concentrated using Amicon Ultra-15 (Millipore, Billerica, MA, USA) and protein quantification was evaluated using the Pierce Bicinchoninic Acid Protein Assay Kit (Thermo Scientific). A mono-specific polyclonal hyperimmune serum was produced in rabbits using the purified $\mathrm{Zmp}$ protein as previously described [25].

\section{IgA protease cleavage assay}

The IgA protease cleavage assay was performed as previously described with some modifications $[10,14]$. Briefly, $5 \mu \mathrm{g}$ of human myeloma IgA (Calbiochem/EMD Millipore, San Diego, CA, USA) were incubated with either purified recombinant Zmp $(100 \mu \mathrm{g}), 1 \times 10^{8} \mathrm{CFU} /$ $\mathrm{mL}$ of live washed $S$. pneumoniae (positive control), $1 \times 10^{9} \mathrm{CFU} / \mathrm{mL}$ of live washed S. suis $\mathrm{P} 1 / 7$ strain or supernatants (non-concentrated or concentrated up to 10X) of each bacterial species for $16 \mathrm{~h}$ at $37{ }^{\circ} \mathrm{C}$. Using similar conditions, the clonal ST7 strain SC84, responsible for the 2005 Chinese human outbreak [26], was also tested. Bacterial supernatants were filtrated and concentrated up to 10X using the Thermo Savant DNA120 Speedvac (Thermo Fisher). Samples were then separated on $7.5 \%$ SDS-PAGE under denaturing conditions and transferred onto nitrocellulose membrane (Bio-Rad). The size of uncleaved and cleaved human IgA $_{1}$ was detected using Western blot with mouse monoclonal B3506B4 anti-human IgA $\mathrm{A}_{1} \mathrm{Fc}$ horseradish peroxidase (HRP)-conjugated antibodies diluted 1:500 (Abcam, Cambridge, MA, USA) and detected using HyGLO Chemiluminescent HRP Antibody Detection Reagent (Denville Scientific, Metuchen, NJ, USA).

\section{Activation of matrix metalloprotease-9}

MMP-9 activation assay was performed as previously described with some modifications [10,14]. Briefly, $10 \mathrm{ng}$ of recombinant human pro-enzyme MMP-9 (Calbiochem/EMD Millipore) were incubated with recombinant Zmp (100 $\mu \mathrm{g})$, S. suis or S. pneumoniae (washed bacteria and supernatants as described above) for $1 \mathrm{~h}$ at $37^{\circ} \mathrm{C}$. Samples were then separated on $10 \%$ zymogram gels containing gelatin B (Sigma) under non-denaturing conditions. Results were visualized after Coomassie Blue R250 staining (Bio-Rad).

\section{Cleavage of P-selectin glycoprotein ligand-1 ectodomains}

The PSGL-1 cleavage assay was performed as previously described with some modifications [15]. Briefly, $10 \mathrm{ng}$ of recombinant human PSGL-1/CD162 Fc (R\&D Systems, Minneapolis, MN, USA) were incubated with recombinant Zmp (100 $\mu \mathrm{g})$, S. suis or S. pneumoniae (washed 
bacteria and supernatants, as described above) for $1 \mathrm{~h}$ at $37{ }^{\circ} \mathrm{C}$. Samples were then separated on $7.5 \%$ SDS-PAGE under denaturing conditions and transferred to polyvinyl difluoride (PVDF) membrane (EMD Millipore). The size of cleaved and uncleaved human PSGL-1/Fc was detected using Western blot with mouse monoclonal anti-human CD162 antibodies, clones KPL-1 and PL2, diluted 1:1000 (Becton-Dickinson Biosciences; MBL International Corporation, Woburn, MA, USA) and horseradish peroxidase conjugated (HRP) goat anti-mouse antibodies (Jackson ImmunoResearch, West Grove, PA, USA). Detection was achieved by using HyGLO Chemiluminescent HRP Antibody Detection Reagent (Denville Scientific).

\section{Cleavage of mucin 16 ectodomains}

The MUC16 ectodomain cleavage assay was performed as previously described with some modifications [13, 27]. The HeLa (CCL-2) cell line was obtained from the ATCC and incubated at $37{ }^{\circ} \mathrm{C}$ with $5 \% \mathrm{CO}_{2}$. Once confluent, cells were trypsinized, transferred to 24-well tissue culture plates (Costar) at a concentration of $7.5 \times 10^{4}$ cells/ $\mathrm{mL}$, and further incubated to confluence from 2 to 3 days (approximately $10^{5}$ cells/well) [28]. Cells were then washed twice with phosphate-buffered saline (PBS), $\mathrm{pH}$ 7.3, before being treated with recombinant $\mathrm{Zmp}(100 \mu \mathrm{g})$, S. suis or S. pneumoniae, which was used as a positive control (washed bacteria and supernatants as described above). In a parallel study, $S$. suis $\Delta z m p$-concentrated (3X) supernatants supplemented or not with $100 \mu \mathrm{g}$ of recombinant $\mathrm{Zmp}$ and $S$. suis comp $\Delta z m p$ concentrated (3X) supernatants were also tested. Absence of toxicity was verified using the CytoTox $96^{\circledR}$ Assay Kit (Promega, Madison, WI, USA). Cells were incubated for $4 \mathrm{~h}$ at $37{ }^{\circ} \mathrm{C}$, after which supernatants were recovered and concentrated two-fold with Thermo Savant DNA120 Speedvac (Thermo Fisher). Cleaved MUC16 ectodomains were determined using DOT-blot on nitrocellulose membrane with 1:100 mouse anti-human CA125 antibody (M11) (Thermo Fisher) and a 1:4000 HRP conjugated goat antimouse antibody (Jackson ImmunoResearch). Detection was achieved by using the HyGLO Chemiluminescent HRP Antibody Detection Reagent (Denville Scientific) and signals analysed using ImageJ.50i software (Wayne Rasband, National Institutes of Health, Bethesda, MD, USA).

\section{Cleavage of syndecan-1 ectodomains}

SDC-1 ectodomains cleavage assay was performed as previously described with some modifications [12, 17, 18]. Briefly, NMuMG cell line, derived from a mouse mammary gland, was propagated as previously described $[12,17,18]$. One-day post-confluent NMuMG cells in 96-well tissue culture plates were washed with culture medium and then incubated with $10 \%$ concentrated filtered supernatants (10X) from an overnight culture of S. suis, S. suis $\Delta z m p, S$. suis comp $\Delta z m p$ for $4 \mathrm{~h}$ at $37^{\circ} \mathrm{C}$. Absence of toxicity was verified as described above. Cells were also incubated with recombinant $\mathrm{Zmp}(100 \mu \mathrm{g})$ or $10 \%$ S. suis $\Delta z m p$-concentrated supernatant (10X) supplemented with $100 \mu \mathrm{g}$ of recombinant Zmp protein. Phorbol 12-myristate 13-acetate (PMA; Sigma) was used as a positive control at a concentration of $1 \mu \mathrm{M}$. After incubation of $4 \mathrm{~h}$, conditioned media were collected and acidified as previously described [12, 17, 18]. Samples were dot-blotted on Immobilon $\mathrm{Ny}+$ (Millipore) and shed ectodomains were quantified by immunoblotting using 281-2 rat anti-mouse CD138 antibodies. The intensity of dots was quantified by ImageJ.50i software.

\section{Adhesion to primary porcine bronchial epithelial cells}

Primary porcine bronchial epithelial cells (PBEC) were isolated from lungs obtained from 5 to 6 month old healthy pigs as previously described [29] and differentiated in air-liquid-interface medium (ALI medium), which consists of 50\% Dulbecco's Modified Eagle Medium (DMEM; Life Technologies) and 50\% Bronchial Epithelial Growth Medium [BEGM; constituted of bronchial epithelial cell basal medium (BEBM; Lonza) supplemented with several required additives (Sigma)], as previously described [30]. After 4 weeks of differentiation, cells were infected from the apical side, as previously described [29], with approximately $10^{7} \mathrm{CFU} /$ filter of S. suis strain P1/7 (WT) or $\Delta z m p$ mutant. Absence of toxicity was verified as described above. Adhesion was analyzed using immunofluorescence microscopy and quantified as previously described [29]. Briefly, this was done semi-quantitatively using a TCS SP5 confocal laserscanning microscope (Leica) equipped with HCX PL APO CS $20 \times 0.7$ DRY UV. Image stacks with a z-distance of $0.5 \mu \mathrm{m}$ per plane were merged and brightness/contrast was adjusted using the ImageJ/Fiji. Area of epithelial cells positive for adherent green fluorescent bacteria was quantified in four randomly selected areas for each treatment using analySIS ${ }^{\circledR} 3.2$ software (Soft Imaging System GmbH, Münster, Germany).

\section{S. suis $\Delta z m p$ virulence evaluation in mouse models of infection}

In a first experiment, 20 six-week-old CD-1 mice (Charles River, Kingston, ON, Canada) were infected via the intranasal route as previously described [31]. One hour prior to infection, mice were anaesthetized via inhalation of isoflurane (Pharmaceutical Partners of Canada, Richmond Hill, ON, Canada) and pre-treated with 12.5 $\mu \mathrm{L}$ of $1 \%$ acetic acid placed in each nostril. Mice were 
then infected with $8 \times 10^{9} \mathrm{CFU}$ of the wild-type strain or $\Delta z m p$ strain $(n=10)$. The inoculum was applied in two drops of $20 \mu \mathrm{L}$ placed in each nostril. Alongside, another 20 six-week-old CD-1 mice (Charles River) were with $3 \times 10^{7} \mathrm{CFU}$ of the wild-type strain or $\Delta z m p$ strain $(n=10)$ via the intraperitoneal route, as previously described [32].

For both experiments, health and behavior were monitored at least thrice daily until $72 \mathrm{~h}$ post-infection and twice thereafter until the end of the experiment (14 days post-infection) for the development of clinical signs of sepsis, such as depression, swollen eyes, rough hair coat, and lethargy. Mice were also monitored for the development of clinical signs of meningitis. Mice were immediately euthanized upon reaching endpoint criteria using $\mathrm{CO}_{2}$ followed by cervical dislocation. No mice died before meeting endpoint criteria and all surviving mice were euthanized as described above at the end of the experiment.

\section{Evaluation of the colonization capacity and virulence of the S. suis $\Delta z m p$ in a porcine model of infection} Four-week old piglets $(n=12)$ from a herd with no recent cases of $S$. suis disease were used. Animals were randomly separated into two rooms upon arrival (6 animals per room) and the trachea and tonsils were swabbed to confirm absence of serotype 2 European-like strains using a multiplex PCR targeting the epf gene that codes for the EF [33], usually absent in North American serotype 2 strains [3], and cps $2 j$ gene, coding for the capsular polysaccharide of serotype 2 strains. The $S$. suis wild-type serotype 2 strain $\mathrm{P} 1 / 7$ (which is epf and cps $2 j$ positive) and $\Delta z m p$-deficient mutant were cultured as previously described [32] to obtain a final concentration of $2 \times 10^{10} \mathrm{CFU} / \mathrm{mL}$. Intranasal infections were carried out as previously described with some modifications [34]. Pigs were inoculated with $2 \mathrm{~mL}$ of $1 \%$ acetic acid per nostril $2 \mathrm{~h}$ prior to infection with $2 \mathrm{~mL}$ per nostril of either the wild-type strain or $\Delta z m p$ mutant. Trachea and tonsils were swabbed using a catheter (Medline, Waukegan, IL, USA) or a brush (Medical Packaging Corporation, Camarillo, CA, USA), respectively, immediately prior to infection, $24 \mathrm{~h}$ after infection, and every 2 days thereafter. Samples were placed in PBS supplemented with $0.1 \%$ bovine serum albumin and immediately cultured. Serial dilutions $\left(10^{0}-10^{-6}\right)$ were plated on Colombia Agar (Oxoid, Hampshire, UK) supplemented with 5\% defibrinated sheep blood (Cedarlane, Burlington, ON, Canada), and Streptococcus Selective Reagent SR0126 (Oxoid). After incubation for $18 \mathrm{~h}$ at $37{ }^{\circ} \mathrm{C}$ with $5 \% \mathrm{CO}_{2}$, plates containing between 30 and 300 colonies were selected. Suspected alpha-hemolytic colonies were enumerated and $10 \mathrm{~S}$. suis-like colonies/plate were sub-cultured in order to perform the epf and cps2j multiplex PCR [33].

Clinical signs of pigs were monitored throughout the experiment. Nine days post-infection, remaining animals were euthanized. Liver, spleen, and brain samples were collected upon euthanasia. All samples were evaluated for carriage of S. suis serotype $2 \mathrm{epf}^{+}$as described above. S. suis serotype 2 strains recovered from euthanized animals infected with the mutant strain were monitored for absence of the $z m p$ gene by PCR.

\section{Statistical analyses}

Data are expressed as mean \pm standard error of the mean (SEM). Significant differences were determined using the t-test, Mann-Whitney Rank sum test or oneway ANOVA, where appropriate. Each test was repeated in at least three independent experiments. For in vivo virulence experiments, survival was analyzed using the LogRank test. $p<0.05$ was considered statistically significant.

\section{Results}

Confirmation of the Zmp-deficient and complemented $S$. suis mutants

A Western blot using a rabbit anti-Zmp serum confirmed production of Zmp protein in the $S$. suis wild-type strain $\mathrm{P} 1 / 7$, which was abrogated following in-frame deletion of the $z m p$ gene in the mutant strain (Figure 1). Complementation of $z m p$ gene (S. suis comp $\Delta z m p$ ) restored production of Zmp, albeit expression was lower than that of the wild-type parent strain (Figure 1). Moreover, gene deletion and complementation had no impact on growth of the mutant in comparison with the wild-type strain (results not shown). The mutant and complemented strains were shown to be well-encapsulated by the hydrophobicity test and the clear positive reaction by

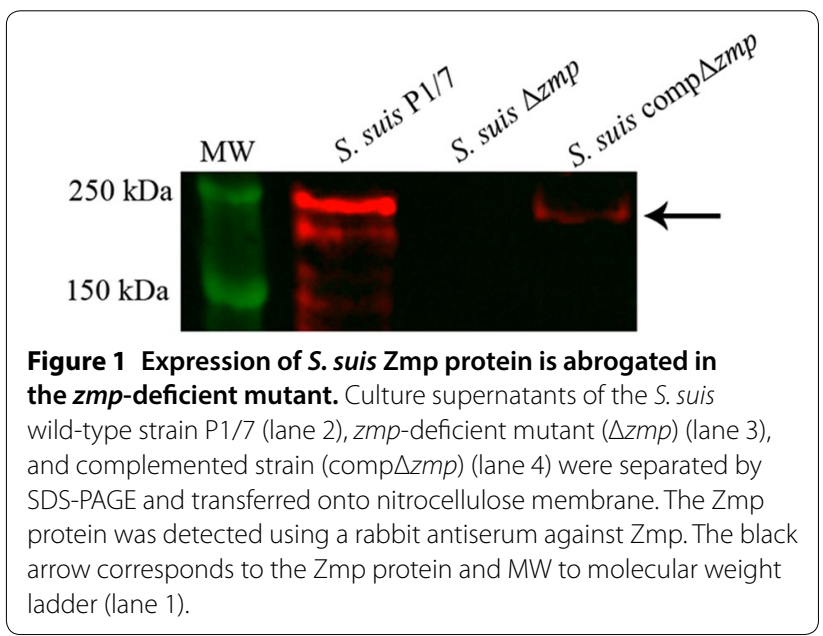


coagglutination using serotype 2 anti-serum (results not shown).

\section{S. suis is unable to cleave human $\lg A_{1}$}

In order to confirm the ability of $S$. suis and Zmp to cleave IgAs, recombinant Zmp, bacterial suspensions (bact) or bacterial supernatants (sup; either non-concentrated or concentrated) were incubated with human $\operatorname{Ig} \mathrm{A}_{1}$. For S. pneumoniae strain TIGR4 (SP), used as a positive control, an IgA protease activity was clearly observed (Figure 2). On the contrary, recombinant S. suis Zmp was unable to cleave human IgAs (Figure 2). In addition, the virulent strain P1/7 of S. suis used in this study (both whole live washed bacteria and culture supernatants) was completely unable to cleave human IgAs (Figure 2). Similarly, S. suis ST7 strain SC84 did not possess any IgA protease activity (Additional file 1).

\section{S. suis does not activate MMP-9}

To assess the capacity of $S$. suis and Zmp to activate MMP-9, the enzyme pro-form (97 kDa) was incubated with recombinant Zmp, bacteria (bact) or bacterial supernatant (sup; either non-concentrated or concentrated). MMP-9 activation was then visualized using gelatin B zymogram, wherein absence of color after Coomassie staining indicates presence of a gelatinase activity. As observed in Figure 3, S. pneumoniae strain TIGR4 (SP), used as a positive control, was able to activate MMP-9 since an additional clear band corresponding to the gelatinolytic activity of activated MMP-9 appeared on the gel at a molecular weight smaller than $82 \mathrm{kDa}$. However, no activation of MMP-9 was observed in the presence of recombinant $\mathrm{Zmp}$ or with S. suis (SS)

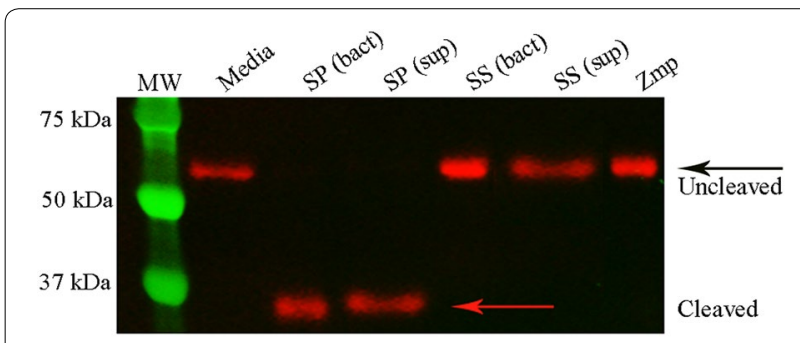

Figure $2 S$. suis is unable to cleave human $\lg A_{1}$. Purified recombinant $Z \mathrm{mp}$ protein $(100 \mu \mathrm{g})$, washed bacteria (bact) or bacterial supernatant (sup) from S. suis (SS) or S. pneumoniae (SP) were incubated with human $\lg \mathrm{A}_{1}$ for $16 \mathrm{~h}$ and reactions separated by SDS-PAGE. Cleaved $\lg \mathrm{A}_{1}$ (red arrow) by washed S. pneumoniae bacteria or bacterial supernatant (lanes 3 and 4) and uncleaved $\lg A_{1}$ (black arrow) by washed S. suis bacteria (lane 5), bacterial supernatant (10X, lane 6), and purified Zmp protein (lane 7) were visualized using a specific antibody against human $\lg \mathrm{A}_{1}$. MW corresponds to the molecular weight ladder (lane 1) and media correspond to untreated human $\lg \mathrm{A}_{1}$ (lane 2).

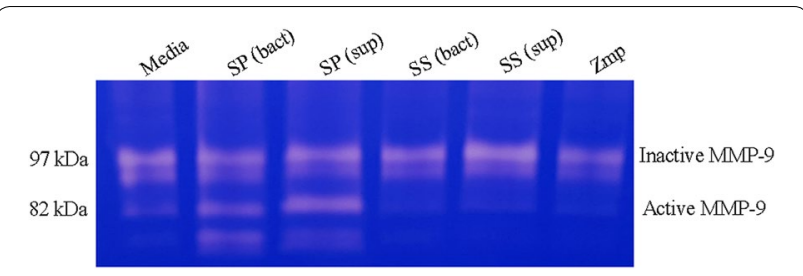

Figure 3 S. suis does not activate matrix metalloprotease-9 (MMP-9). Pro-enzyme MMP-9 was incubated for $1 \mathrm{~h}$ with purified recombinant $Z \mathrm{mp}$ protein $(100 \mu \mathrm{g})$, washed bacteria (bact) or bacterial supernatant (sup) from S. suis (SS) or S. pneumoniae (SP). Samples were separated in non-denaturing conditions on a gelatin B zymogram. Coomassie coloration shows a second gelatinolytic band for washed S. pneumoniae bacteria or bacterial supernatants (lane 2 and 3) corresponding to activated MMP-9, while no second band was observed for media (lane 1) washed S. suis bacteria (lane 4), bacterial supernatant (10X, lane 5) or purified Zmp protein (lane 6).

with either whole live washed bacteria or bacterial supernatants (Figure 3).

\section{S. suis is unable to cleave PSGL-1 ectodomains}

In order to evaluate the ability of S. suis and Zmp to cleave PSGL-1 ectodomains, recombinant $\mathrm{Zmp}$, whole bacteria (bact) or bacterial supernatants (sup) were incubated with PSGL-1/fc. To visualize cleavage, two different antibodies were used, whether KPL-1 and PL-2, which recognize the N-terminal epitope of PSGL-1 and a membrane-proximal epitope, respectively [35]. As expected [15], the S. pneumoniae strain TIGR4 (SP), used as a positive control, cleaved PSGL-1 ectodomains (Figure 4). It was observed that the reactivity of KPL-1 antibody with PSGL-1/fc diminished as a result of the N-terminal epitope of PSGL-1/fc being cleaved (Figure 4A). Meanwhile, PL2 still recognized the degradation products of PSGL-1/Fc (Figure 4B). This was expected since the $S$. pneumoniae $\mathrm{ZmpC}$ removes the $\mathrm{N}$-terminus of PSGL-1 required for recognition by KPL1 with PSGL-1/Fc, since KPL1 recognizes a N-terminal epitope of PSGL-1 [35]. On the other hand, PL2 can still recognize multiple degradation products between 150 and $25 \mathrm{kDa}$ since it recognizes a membrane-proximal epitope [35]. Meanwhile, recombinant Zmp, S. suis (SS) bacterial cells as well as bacterial supernatants were unable to cleave PSGL-1 ectodomains (Figure 4).

\section{Zmp is partially implicated in the capacity of S. suis to cleave MUC16 ectodomains}

A treatment of HeLa cells with live washed bacteria or bacterial supernatants concentrated $10 \mathrm{X}$ was found to be cytotoxic (results not shown). Thus, S. pneumoniae nonconcentrated supernatant (positive control) and S. suis supernatant (either non-concentrated or concentrated 

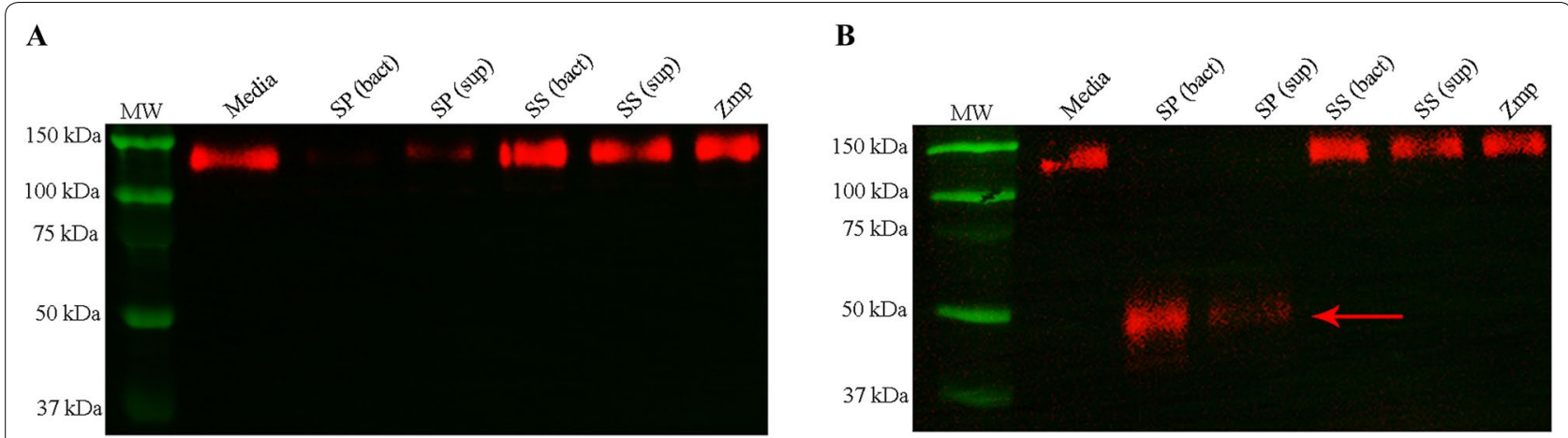

Figure 4 S. suis is unable to cleave P-selectin glycoprotein ligand-1 (PSGL-1) ectodomains. Purified recombinant Zmp protein (100 $\mu \mathrm{g})$, washed bacteria (bact) or bacterial supernatant (sup) from S. suis (SS) or S. pneumoniae (SP) were incubated with PSGL-1/fc for 1 h. Samples were separated by SDS-PAGE and transferred onto PVDF membranes, which were incubated with KPL-1 (A) or PL2 (B) antibody. No recognition of PSGL-1 by the KPL-1 antibody and visualization of lower molecular weight band with PL2 antibody were observed for washed S. pneumoniae bacteria and bacterial supernatant (lanes 3 and 4), which is expected following PSGL-1 ectodomain cleavage. There was no change in the recognition by KPL-1 or in the molecular weight visualized with PL2 for PSGL-1 treated with washed S. suis bacteria (lane 5), bacterial supernatant (10X, lane 6) or the purified recombinant Zmp protein (lane 7). MW corresponds to the molecular weight ladder (lane 1) and media correspond to untreated PSGL-1 (lane 2).

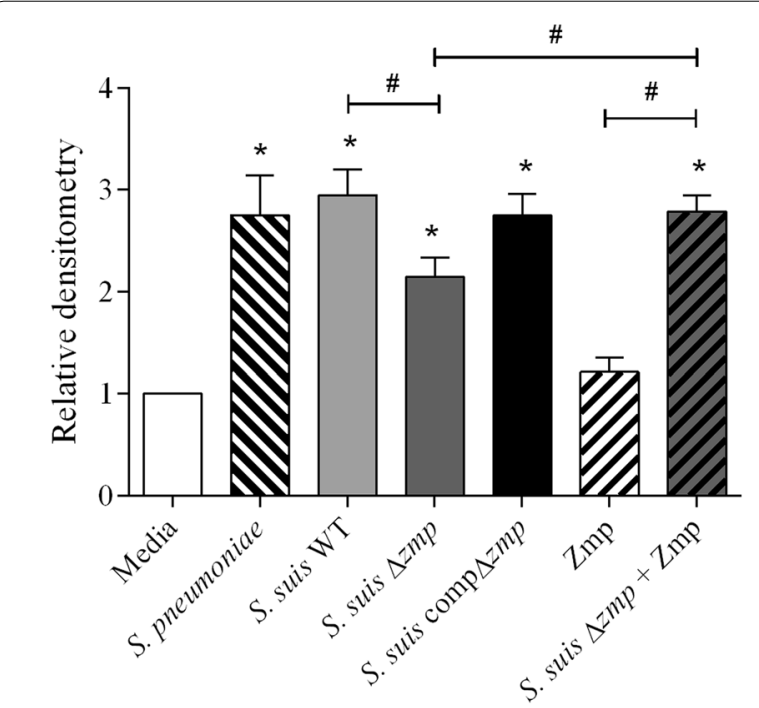

Figure $5 \mathrm{Zmp}$ is partially implicated in mucin 16 (MUC16) ectodomain cleavage by $S$. suis. Relative densitometry of HeLa cell culture medium conditioned with non-concentrated S. pneumoniae supernatant, Zmp purified recombinant protein, S. suis P1/7 (WT) or S. suis $\triangle z m p$ supernatant (3X) supplemented or not with $100 \mu \mathrm{g}$ of recombinant Zmp protein. Samples were blotted on a nitrocellulose membrane and incubated with anti-human CA125 antibodies. Compared to the untreated cells, S. suis WT strain P1/7 supernatant cleaved MUC16 ectodomains. This activity was significantly reduced when using the supernatant of the $\Delta z m p$ strain $(p=0.017)$. Supernatant of the comp $\Delta z m p$ significantly increased cleavage when compared to the $\Delta z m p$ strain. Addition of $100 \mu \mathrm{g}$ of $Z m p$ protein to the supernatant of the $\triangle z m p$ strain also restored WT phenotype activity. Data represent the mean \pm SEM from at least three independent experiments. ${ }^{*}(p<0.05)$ indicates a significant difference between treated groups and the medium; ${ }^{*}(p<0.05)$ between treatment groups.
3X) were used to perform the MUC16 ectodomain cleavage assay. As shown in Figure 5, S. pneumoniae non-concentrated supernatant cleaved MUC16 ectodomains. However, only after being concentrated $3 \mathrm{X}$ was $S$. suis supernatant from wild-type strain P1/7 able to cleave MUC16 ectodomains. As such, Zmp activity of S. suis seems to be less strong than that of $S$. pneumoniae. Furthermore, deletion of the $z m p$ gene significantly decreased the ability of $S$. suis supernatant to cleave MUC16 ectodomains $(p=0.017)$, but not to the level of the negative control. However, S. suis recombinant Zmp alone is unable to cleave MUC16 ectodomains $(p=0.304)$. Meanwhile, complementation of $z m p$ gene in S. suis (comp $\Delta z m p)$ partially restored the wildtype phenotype, in comparison with the isogenic mutant $(p=0.067)$, but was still less active than the wild-type strain. However, complementation of the S. suis $\Delta z m p$ supernatant by addition of $100 \mu \mathrm{g}$ of recombinant $\mathrm{Zmp}$ completely restored the wild-type phenotype $(p=0.907)$. Residual MUC16 ectodomain shedding ability when using the $S$. suis $\Delta z m p$ supernatant suggests that other factors are potentially responsible for cleavage by $S$. suis.

\section{Zmp is partially implicated in the capacity of $S$. suis to cleave SDC-1 ectodomains}

NMuMG cells treated with $100 \mu \mathrm{g}$ of recombinant Zmp or S. suis bacterial supernatants were used to assess SDC-1 ectodomain cleavage. Supernatant concentrated 10X (but not non-concentrated or concentrated 3X; data not shown) from S. suis wild-type strain P1/7 was able to cleave SDC-1 ectodomains (Figure 6). Furthermore, deletion of the $z m p$ gene significantly decreased this ability 


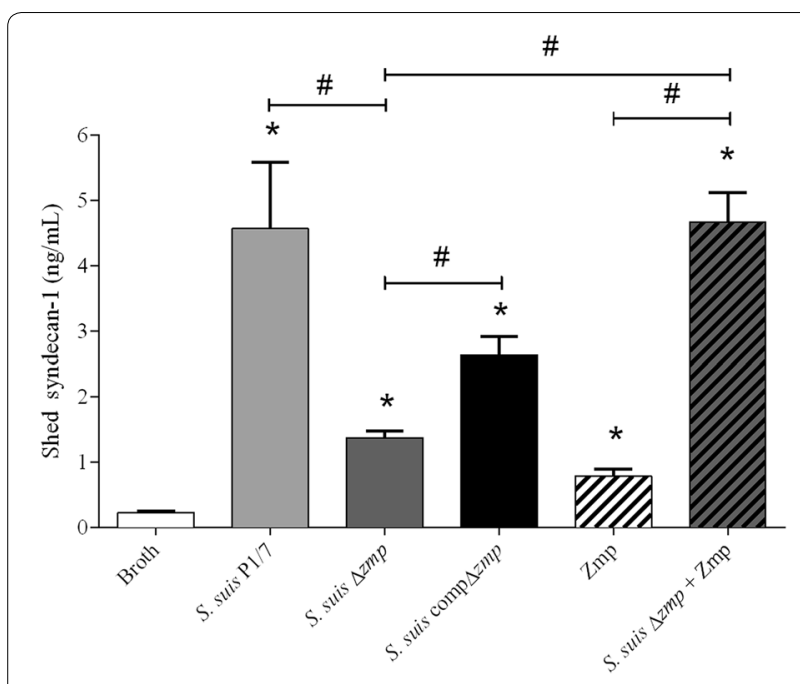

Figure $6 \mathrm{Zmp}$ is implicated in SDC-1 ectodomain cleavage. The concentration of SDC-1-cleaved ectodomains in NMuMG cell culture medium was measured after treatment with $100 \mu \mathrm{g}$ of Zmp protein, 10X concentrated supernatants of either S. suis wild-type P1/7 (WT), S. suis $\triangle z m p$ (supplemented or not with $100 \mu \mathrm{g}$ of recombinant Zmp) or S. suis comp $\Delta z m p$. Samples were blotted on Immobilon $\mathrm{Ny}+$ and incubated with anti-mouse SDC-1 (281-2) antibodies. The capacity of the $\triangle z m p$ mutant to cleave SDC-1 ectodomains was significantly reduced in comparison to the WT strain, while the complementation of zmp gene restored WT phenotype. Recombinant Zmp protein also cleaved SDC-1 ectodomains. This activity was amplified when Zmp protein was incubated with zmp-deficient mutant culture supernatant. The concentration of shed SDC-1 ectodomains was determined using SDC-1 ectodomains purified from NMuMG cells as standards, as previously described [12]. Data represent the mean \pm SEM from at least three independent experiments. ${ }^{*}(p<0.05)$ indicates a significant difference between treated groups and the medium; ${ }^{\#}(p<0.05)$ between treated groups. using the same supernatant concentration $(p=0.024)$; the SDC-1 cleavage activity was restored by complementation of $z m p$ gene in strain S. suis (comp $\Delta z m p)(p=0.030)$, when compared to the activity of the $\Delta z m p$ mutant strain (Figure 6). Moreover, even if the level of SDC-1 ectodomains released was lower than that by the wild-type strain, there was no statistical difference between these two groups $(p=0.139)$. In contrast to MUC16 ectodomain shedding experiments, $S$. suis recombinant Zmp was also able to cleave SDC-1 ectodomains when used alone $(p=0.007)$ (Figure 6). Moreover, addition of $100 \mu \mathrm{g}$ of purified recombinant Zmp protein to S. suis $\Delta z m p$ mutant supernatant completely restored wild-type phenotype $(p=0.024)$, suggesting, once again, the presence of co-factor(s) in S. suis supernatant. There was also a residual SDC-1 shedding ability in the $z m p$-deficient mutant, suggesting that other factors could potentially be also involved in this activity.

\section{Zmp is not implicated in adhesion to differentiated primary PBEC}

The ability of the S. suis wild-type strain P1/7 and S. suis $\Delta z m p$ mutant to colonize primary porcine bronchial epithelial cells was analyzed in ALI cultures with primary PBEC. Cells were infected with $10^{7} \mathrm{CFU} /$ filter (approximate $\mathrm{MOI}=25$ ) from the apical side for $4 \mathrm{~h}$ and non-adherent bacteria were washed away. Immunofluorescence microscopy showed that the wild-type and $\Delta z m p$ strains similarly adhered to ciliated epithelial cells (Figures 7A and B). Quantification of adherent bacteria revealed no significant differences in the ability of both strains to colonize PBEC (Figure 7C).
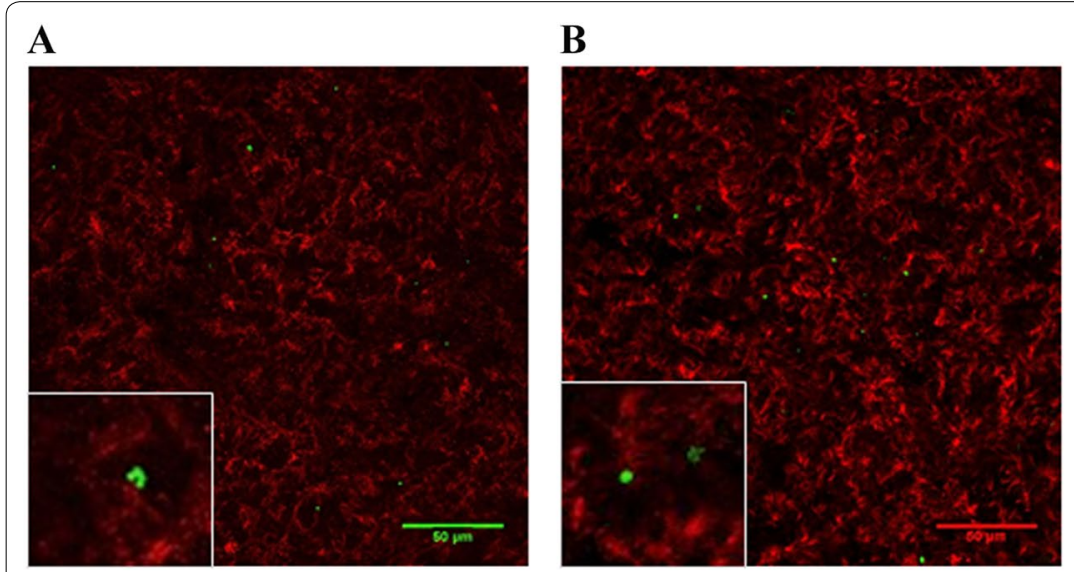

C

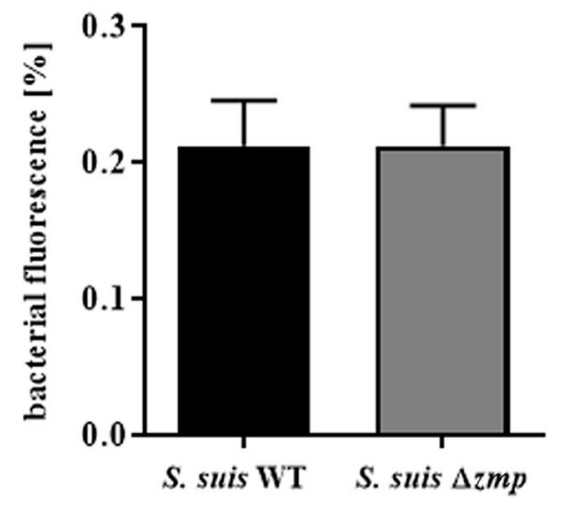

Figure 7 Zmp is not implicated in adhesion to PBEC under ALI conditions. Immunofluorescence analyses of PBEC differentiated under ALI conditions and infected for $4 \mathrm{~h}$ with $\mathbf{A}$ S. suis wild-type P1/7 (WT) and B S. suis $\Delta z m p$ strain. Ciliated cells were stained for B-tubulin (red) and streptococci (green). Bars represents $50 \mu \mathrm{m}$. C Adherent bacteria were quantified by analyzing the epithelial cell surface positive for green fluorescence signal in four randomly chosen areas for each treatment. Results are expressed as mean \pm SEM. 
Zmp is not implicated in colonization of the upper respiratory tract of pigs nor in virulence

Considering the presence of MUC16 in the upper respiratory tract of pigs, and given the previously described function of SDC-1 in adhesion to and invasion of epithelial cells [36, 37], the role of Zmp in the pathogenesis of S. suis serotype 2 infection, more particularly in colonization, as well as its role as a virulence factor, were evaluated using an intranasal piglet model of S. suis infection. Animals were divided into two groups and infected with either wild-type strain $\mathrm{P} 1 / 7$ or its $z m p$-deficient mutant. After infection, PCR tests showed presence of $S$. suis serotype 2 ST1 $(c p s+, e p f+)$ in infected animals. As illustrated in Figure 8A, tonsil colonization was already detectable $24 \mathrm{~h}$ post-infection in both groups. As shown in Figure 8B, S. suis serotype 2 ST1 was also present in the trachea of almost all infected animals in both groups and remained present until day 9. Throughout the experiment, no significant differences were observed between wild-type and $\Delta z m p$-infected piglets in both the tonsils and trachea (Figures 8A and B). In addition, results showed no differences in virulence in pigs, since several animals presented severe clinical signs such as arthritis, depression, and meningitis in both groups $(p=0.840)$ (Table 3). Moreover, 33\% of piglets in both groups succumbed to infection (Figure 9). Absence of $z m p$ gene was confirmed in all $S$. suis serotype 2 bacteria recovered from the liver, spleen, and brain of ill animals infected with the mutant strain data not shown). Similar results were also obtained in mice following intranasal $(p=0.976)$ and intraperitoneal $(p=0.346)$ infections
Table 3 Clinical signs observed in wild-type (WT)- and $\Delta z m p$-infected pigs

\begin{tabular}{lll}
\hline Clinical signs & WT & $\boldsymbol{\Delta z m p}$ \\
\hline Fever & $4 / 6$ & $4 / 6$ \\
Respiratory problems & $0 / 6$ & $1 / 6$ \\
Arthritis & $3 / 6$ & $5 / 6$ \\
Neurological problems & $2 / 6$ & $2 / 6$ \\
\hline
\end{tabular}

(Additional file 2). Following intranasal infection, only $20 \%$ of mice presented severe clinical signs, including prostration, depression, and weakness (Additional file 2A). Meanwhile, all intraperitoneally inoculated mice developed severe clinical signs of infection within 5 days post-infection (Additional file 2B).

\section{Discussion}

Increased research over the past decade has led to the identification of a myriad of novel factors putatively involved in the first steps of the S. suis pathogenesis of infection. In many cases, newly identified factors have been described as "critical" for virulence based on little supporting experimental data, and in some cases, controversial results have been reported in subsequent investigations [7]. One example is the $\mathrm{Zmp}$ protease encoded by gene iga $[8,10,38]$. Zinc-dependent metalloproteases have been studied relatively extensively in $S$. pneumoniae. Four distinct groups have been described: $\mathrm{ZmpA}, \mathrm{ZmpB}$, and ZmpC and ZmpD [10]. S. pneumoniae ZmpA has been shown to cleave human IgAs, which
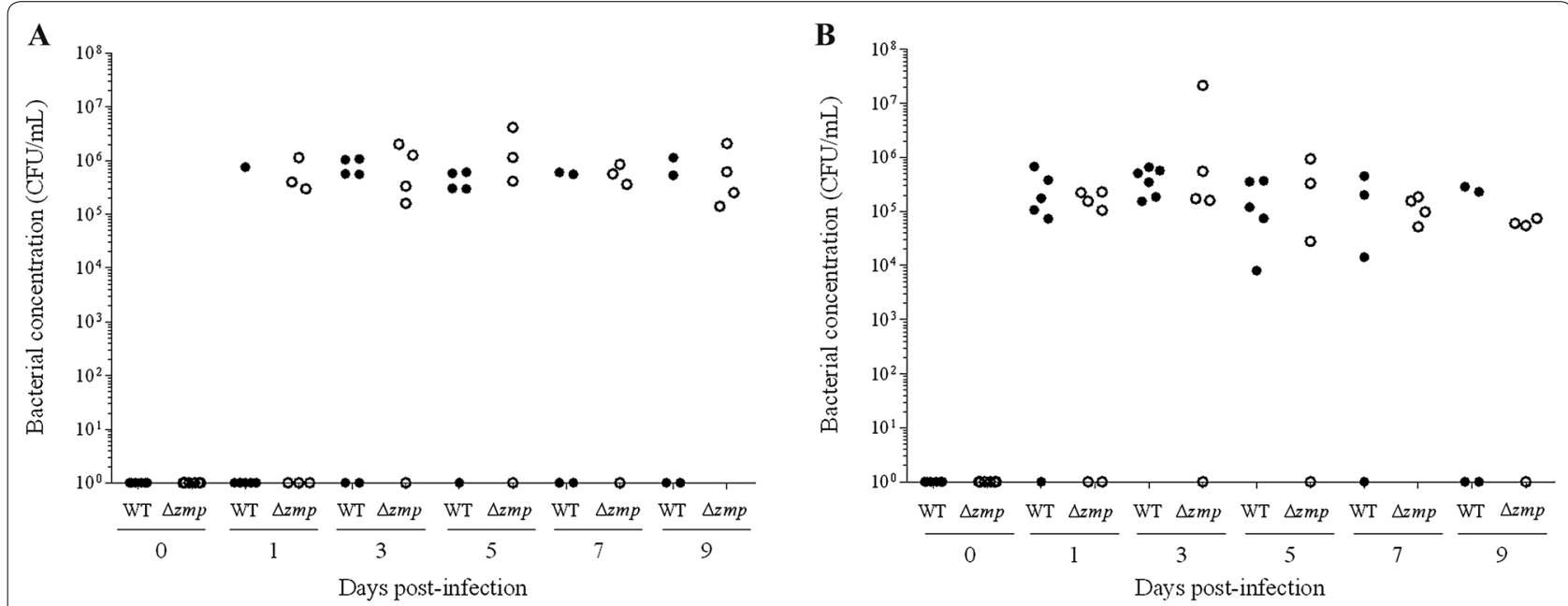

Figure 8 Presence of $\mathrm{Zmp}$ does not influence the colonization potential of $S$. suis serotype 2 in a pig model of infection. Bacterial concentrations recovered in the tonsils $(\mathbf{A})$ or trachea $(\mathbf{B})$ of piglets infected with either the wild-type strain P1/7 (WT) or its $\Delta z m p$ mutant. No differences in colonization of the upper respiratory tract of piglets were observed following infection with the $\Delta z m p$ mutant. Each point represents an individual animal. 


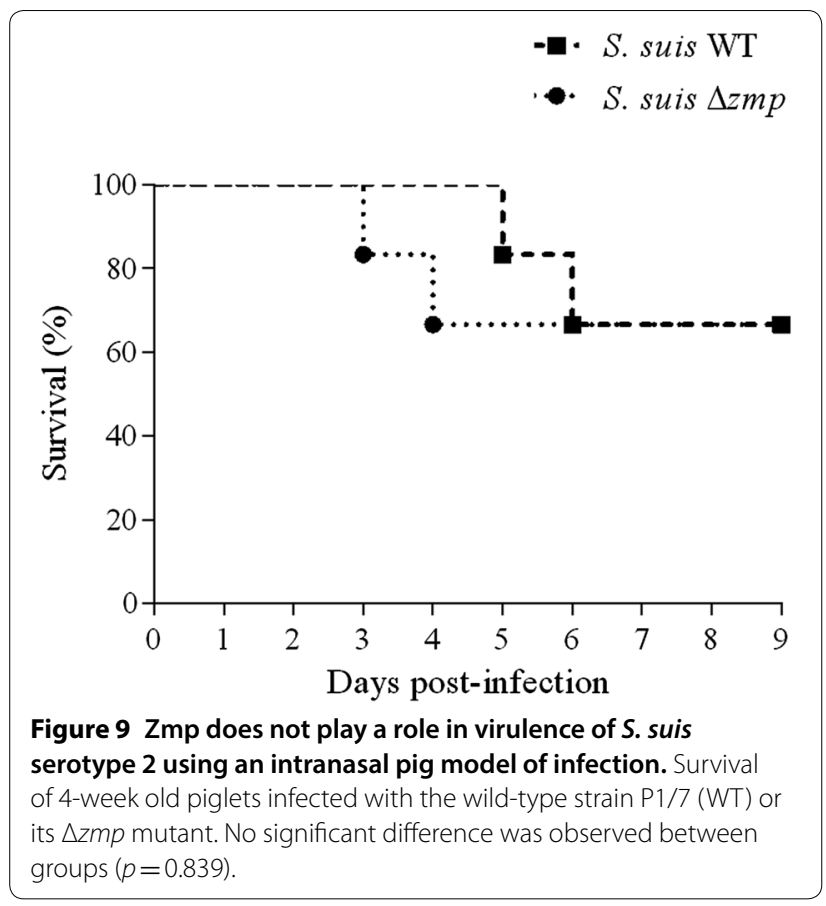

play an important role in the immunological defense of the respiratory tract and other mucosal surfaces [5], with $\operatorname{Ig} \mathrm{A}_{1}$ representing $90 \%$ of the IgA within the human respiratory tract [39]. Indeed, $\operatorname{IgA}_{1}$ proteases have been regarded as virulence factors in a variety of pathogens [39-41]. In S. suis, an IgA protease, with active cleavage effect on human Ig $\mathrm{A}_{1}$, has been previously described in a virulent S. suis isolate [8]. In addition, an isogenic mutant defective for the gene encoding this IgA protease was shown to be significantly less virulent than the wild-type strain. Although IgA protease activity of the mutant or parent strain was not experimentally tested, the animal data were interpreted to mean that IgA cleavage in vivo was crucial for virulence [38]. Later, it was shown that immunization with this IgA protease resulted in 100\% protection of mice against challenge with a virulent strain [42].

Surprisingly, in a comparative study using different streptococci, Bek-Thomsen et al. reported absence of IgA protease activity in the seven S. suis strains tested [10]. Consequently, given the controversy surrounding the activity of this metalloprotease in S. suis, the capacity of S. suis serotype 2 strain $\mathrm{P} 1 / 7$ (ST1) to degrade human $\mathrm{IgA}_{1}$ was evaluated. Results obtained in the present study contradict those originally reported [8] and confirm those published later by Bek-Thomsen et al. [10], with a total absence of IgA protease activity from both S. suis (non-concentrated or concentrated live washed bacteria/ bacterial supernatants) and the cloned, expressed, and purified recombinant protein. Both the reference virulent ST1 strain, as well as the highly virulent ST7 strain SC84 (which corresponds to the strain used in the original report by Zhang et al. [8]) presented identical results. These results also confirm the larger controversy regarding critical S. suis virulence factors $[7,43]$.

The study of Bek-Thomsen et al. also suggested that the S. suis $\mathrm{Zmp}$ homologue belongs to the $\mathrm{ZmpC}$ subgroup [10], and comparison of the S. suis serotype 2 Zmp sequence with that of S. pneumoniae showed $42 \%$ of homology between both proteins (Additional file 3). Moreover, the S. suis $\mathrm{Zmp}$ was also previously described to possess a HEMVH motif in its active site, which corresponds to that of $\mathrm{ZmpC}[8,10]$. Regardless of these genetic similarities, the specific properties and functions of the S. suis Zmp protein remained unknown. S. pneumoniae ZmpC activates the pro-form of MMP-9 and cleaves PSGL-1, MUC16, and SDC-1 ectodomains [12, $14,15,27]$.

Firstly, the involvement of the S. suis Zmp in activation of MMP-9 by pro-form cleavage was evaluated [14]. Of the different MMPs described, MMP-9 has been largely associated with the disruption of both the blood-brain barrier and the blood-cerebrospinal fluid barrier [16]. Given that one of the most important pathologies caused by $S$. suis in diseased pigs is meningitis, MMP-9 could play an important role in the infection [1]. Previous studies have demonstrated that $S$. suis serotype 2 induces production of MMP-9 from macrophages, and it was suggested that this production might help the pathogen to invade the CNS $[44,45]$. Results from this study show that, under the conditions tested, and differently from S. pneumoniae, recombinant Zmp does not activate the pro-enzyme form of MMP-9. Moreover, we did not identify this activity in S. suis serotype 2 . Thus, S. suis appears to behave similarly to Streptococcus sanguinis and Streptococcus oralis, which are also unable to activate MMP-9, despite possessing a $z m p C$ gene encoding putative metalloproteases with an HEMTH motif in their active site [10]. We speculate that previously described MMP-9 activation in macrophages during interactions with S. suis may instead be due to internal control mechanisms of the host, such as the secretion of plasmin and elastase by host cells [46, 47].

PSGL-1 is an important molecule expressed at the surface of certain immune cell types and, during stress conditions, it binds the P-selectin at the surface of endothelial cells, which will subsequently allow the diapedesis of cells from the blood vessels to the site of infection [48]. Numerous pathogens, including S. pneumoniae, have thus developed strategies to outmaneuver the immune system, including cleavage of PSGL-1 [15, 48]. Results obtained in this study showed that, unlike 
that of S. pneumoniae, S. suis serotype 2 and its Zmp protein are unable to cleave PSGL-1 ectodomains.

Next, the ability of S. suis and its Zmp to cleave MUC16 was evaluated. MUC16 is a membrane-associated mucin that is part of the epithelial barrier, and it is found, among other mucosal surfaces, at the respiratory tract levels of mammals $[49,50]$. S. pneumoniae, via its $\mathrm{ZmpC}$ protein, could disrupt the epithelial barrier by cleavage of MUC16 ectodomains, allowing the pathogen to more easily colonize host epithelial cells and underlying basement membranes $[13,27]$. In this study, results showed that $S$. suis serotype 2 liberates the MUC16 ectodomains and that this function is Zmp-dependent. This could play a role in S. suis adhesion to and invasion of epithelial cells, as was shown for Staphylococcus aureus [51]. However, the observed activity seems to be of lower intensity that that of S. pneumoniae, as the S. suis supernatant induced this effect only after being concentrated. In addition, and differently from what was reported for S. pneumoniae [27], recombinant S. suis $\mathrm{Zmp}$ alone was not able to induce the cleavage of MUC16 ectodomains. Indeed, recombinant S. suis Zmp protein seems to require a co-factor found in the bacterial supernatant in order to be active. This co-factor could be a potential cation or protease: further studies are needed to examine these hypotheses in detail. In addition, S. suis has numerous virulence factors described with redundant functions that could compensate for the loss of another factor [6].

Cleavage of SDC-1 ectodomains was then investigated [12]. SDC-1 is a type 1 transmembrane heparan sulfate proteoglycan mainly expressed by plasma cells and epithelial cells and, to a lesser extent, endothelial cells, macrophages, and fibroblasts [37, 52]. SDC1 (CD138) is used as a marker for plasma cells, and myeloma cells and several carcinomas, such as breast cancer cells, express high levels of SDC1. Interactions of bacterial pathogens with heparan sulfate proteoglycans such as SDC-1 have been described as important steps in the pathogenesis of different infections $[36,53]$. In accordance, the liberation of SDC- 1 ectodomains by $\alpha$ and $\beta$-toxins, allows $S$. aureus to avoid bacterial killing by neutrophils. This could help pathogens to persist in the circulation more easily [54]. Results obtained in the present study confirm that $S$. suis induces SDC-1 shedding, with this activity mainly being Zmp-dependant. Interestingly, a synergic activity was shown when $\mathrm{Zmp}$ protein was incubated with $S$. suis $\Delta z m p$ supernatant, supporting the hypothesis that the activity of $\mathrm{Zmp}$ requires a co-factor present in the $S$. suis supernatant. Moreover, as was observed with MUC16, a residual SDC-1 ectodomain shedding activity remains in S. suis $\Delta z m p$ strain, suggesting that other factor(s) could also be implicated in this function. Taken together, these functional assay results demonstrate that the $S$. suis serotype $2 \mathrm{Zmp}$ protein does not possess all of the functions of the S. pneumoniae ZmpC.

Given that S. suis cleaved MUC16 ectodomains and the fact that the Zmp protein plays an important role in this function, its role in the adhesion to differentiated primary porcine bronchial cells was investigated. ALI culture with PBEC represents an in vitro model of well-differentiated ciliated and mucus-producing respiratory epithelial cells [29]. Since MUC16 is a mucin recovered in the mucosal barrier of respiratory epithelial cells, PBEC are a good model to evaluate the role of $S$. suis serotype $2 \mathrm{Zmp}$. However, both $S$. suis wild-type and $\Delta z m p$ strains had a similar capacity to adhere to PBEC under ALI conditions. This lack of difference was confirmed in vivo. Regardless of its role in MUC16 ectodomain cleavage in vitro, no difference was observed between the wild-type and the $z m p$-deficient mutant strains in colonization of tonsils and trachea by S. suis after bacterial challenge via the intranasal route. The lack of a critical role in epithelial cell adhesion and colonization might be explained by the concept of bacterial redundancy, which is very common in S. suis [7]. In fact, this result is not necessarily surprising given the number of virulence factors expressed by S. suis serotype 2 that have been reported to be implicated in colonization of the upper respiratory tracts of pigs, including adhesins and toxins. Indeed, around 40 different factors have been described to be involved in S. suis colonization [6]. Animals infected with either the wild-type or the $\Delta z m p$ strain also presented similar clinical signs and mortality rate. This indicates that a lower liberation of SDC-1 ectodomains by the S. suis $\Delta z m p$ mutant did not increase bacterial killing in vivo. As mentioned above, different anti-phagocytic bacterial factors have been described as playing important roles during S. suis infection [7].

Finally, and differently from what has been observed in a previous study [38], where the S. suis IgA protease encoded by iga gene was characterized as a critical virulence factor using an isogenic iga mutant (equivalent to the S. suis $\Delta z m p$ strain used herein), the S. suis $\Delta z m p$ strain was as virulent as the wild-type strain in an pig intranasal model of infection. In the case of S. pneumoniae, results differed regarding the impact of $\mathrm{ZmpC}$ in virulence. While a mutant $\triangle z m p C$ seems to have a reduced virulence in an intranasal mouse model of infection [14, 53], the $\Delta z m p C$ mutant showed an exacerbate virulence compared to the wild-type strain in an intravenous model of infection [55]. This does not seem to be the case for S. suis, since no significant difference was observed in virulence after intraperitoneal infection of mice with the wild-type and $z m p$-deficient mutant strains. 
In conclusion, absence of an $\operatorname{Ig} \mathrm{A}_{1}$ protease activity in $S$. suis serotype 2 was confirmed. Indeed, the $S$. suis metalloprotease hypothetically responsible for such activity would rather belong to the $\mathrm{ZmpC}$ family reported for $S$. pneumoniae. The iga terminology for the gene coding for the Zmp of S. suis should be avoided and changed by $z m p c$. Of the different functions previously described for streptococcal $\mathrm{ZmpC}$, that of S. suis would be responsible for a partial ability to cleave MUC16 and SDC-1 ectodomains. As such, the present study is the first to show such capacities for S. suis. However, the presence of this protein does not appear to be critical for colonization of the porcine upper respiratory tract. In addition, and in disagreement with published results, this protein would not be a critical virulence factor. These results further emphasize, as recently suggested, the need to confirm the critical role of reported candidates in virulence by independent laboratories $[7,43]$.

\section{Additional files}

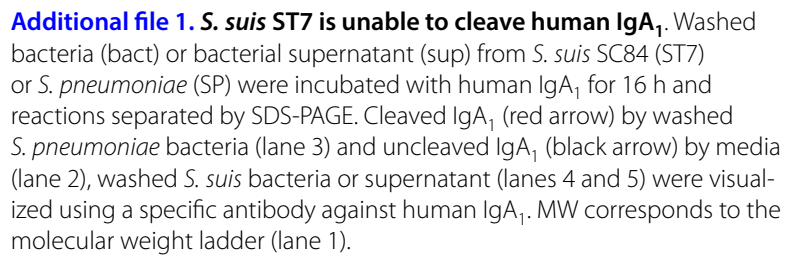

Additional file 2 . $Z \mathrm{mp}$ is not implicated in the virulence of $S$. suis serotype 2 in mouse models of infection. Survival of six-week-old CD-1 mice infected with the wild-type strain P1/7 (WT) or its $\Delta z m p$ mutant via (A) the intranasal or (B) the intraperitoneal route of infection. No significant differences were observed between groups.

\section{Additional file 3. S. suis $\mathrm{Zmp}$ and S. pneumoniae $\mathrm{ZmpC}$ amino} acid sequence alignment. Alignment was performed using T-Coffee. Conserved amino acid appear in gray and identical amino acid appear in black

\section{Abbreviations}

ALI: air-liquid-interface; BCA: bicinchoninic acid; BEBM: bronchial epithelial cell basal medium; BEGM: bronchial epithelial growth medium; CPEC: circular polymerase extension cloning; DMEM: Dulbecco's modified eagle medium; HRP: horseradish peroxidase; IgA: immunoglobulin A; IPTG: isopropyl- $\beta$-Dthiogalactopyranoside; LB: Luria-Bertani; MMP-9: matrix metalloproteinase 9; MUC16: mucin 16; PBEC: porcine bronchial epithelial cells; PBS: phosphatebuffered saline; PCR: polymerase chain reaction; pi: post-infection; PMA: phorbol-12-myristate-13-acetate; PSGL-1: P-selectin glycoprotein ligand-1; SDC-1: syndecan-1; SEM: standard error of the mean; ST: sequence type; THA: Todd-Hewitt agar; THB: Todd-Hewitt broth; WT: wild-type; Zmp: zinc metalloprotease.

\section{Competing interests}

The authors declare that they have no competing interests.

\section{Authors' contributions}

Conception of the work: AD, PVW, PWP, NF, JH, MG; laboratory techniques: AD, JPA, DR, DV, MW, PWP; acquisition, analysis and interpretation of data: AD, JPA, $D R, D V, M W, P V W, P W P, D G, N F, J H, M G$; preparation of the manuscript: AD, JPA, MG. All authors read and approved the final manuscript.

\section{Acknowledgements}

The authors would like to thank Sonia Lacouture (University of Montreal) for invaluable technical assistance.

\section{Author details}

${ }^{1}$ Swine and Poultry Infectious Diseases Research Center (CRIPA), Department of Pathology and Microbiology, Faculty of Veterinary Medicine, University of Montreal, Saint-Hyacinthe, QC, Canada. ${ }^{2}$ Groupe de recherche sur les maladies infectieuses en production animale (GREMIP), Department of Pathology and Microbiology, Faculty of Veterinary Medicine, University of Montreal, Saint-Hyacinthe, QC, Canada. ${ }^{3}$ Institute for Microbiology, University of Veterinary Medicine Hannover, Hannover, Germany. ${ }^{4}$ Boston Children's Hospital, Harvard Medical School, Boston, MA, USA. ${ }^{5}$ Oral Ecology Research Group, Faculty of Dentistry, Laval University, Quebec City, QC, Canada. ${ }^{6}$ Public Health Ontario Laboratory Toronto, and Department of Laboratory Medicine and Pathobiology, University of Toronto, Toronto, ON, Canada.

\section{Availability of data and materials}

The data and materials not presented in this manuscript are available from the corresponding author upon request.

\section{Ethics approval and consent to participate}

All experiments involving animals were conducted in accordance with the guidelines and policies of the Canadian Council on Animal Care and the principles set forth in the Guide for the Care and the Use of Laboratory Animals by the Animal Welfare Committee of the University of Montreal, which approved the protocols and procedures used herein (Permit Number RECH-1570). A total of 12 pigs were used in this study.

\section{Funding}

This study was funded by the Natural Sciences and Engineering Research Council of Canada (Grant \#04435) to MG. JPA is the recipient of an Alexander Graham Bell Graduate Scholarship_Doctoral Program from NSERC.

\section{Publisher's Note}

Springer Nature remains neutral with regard to jurisdictional claims in published maps and institutional affiliations.

Received: 21 August 2018 Accepted: 16 October 2018

Published online: 29 October 2018

\section{References}

1. Goyette-Desjardins G, Auger JP, Xu J, Segura M, Gottschalk M (2014) Streptococcus suis, an important pig pathogen and emerging zoonotic agent-an update on the worldwide distribution based on serotyping and sequence typing. Emerg Microbes Infect 3:e45

2. Okura M, Osaki M, Nomoto R, Arai S, Osawa R, Sekizaki T, Takamatsu D (2016) Current taxonomical situation of Streptococcus suis. Pathogens 5:E45

3. Fittipaldi N, Xu J, Lacouture S, Tharavichitkul P, Osaki M, Sekizaki T, Takamatsu D, Gottschalk M (2011) Lineage and virulence of Streptococcus suis serotype 2 isolates from North America. Emerg Infect Dis 17:2239-2244

4. Ye C, Bai X, Zhang J, Jing H, Zheng H, Du H, Cui Z, Zhang S, Jin D, Xu Y, Xiong Y, Zhao A, Luo X, Sun Q, Gottschalk M, Xu J (2008) Spread of Streptococcus suis sequence type 7, China. Emerg Infect Dis 14:787-791

5. Segura M, Calzas C, Grenier D, Gottschalk M (2016) Initial steps of the pathogenesis of the infection caused by Streptococcus suis: fighting against nonspecific defenses. FEBS Lett 590:3772-3799

6. Fittipaldi N, Segura M, Grenier D, Gottschalk M (2012) Virulence factors involved in the pathogenesis of the infection caused by the swine pathogen and zoonotic agent Streptococcus suis. Future Microbiol 7:259-279

7. Segura M, Fittipaldi N, Calzas C, Gottschalk M (2017) Critical Streptococcus suis virulence factors: are they all really critical? Trends Microbiol 25:585-599

8. Zhang A, Mu X, Chen B, Liu C, Han L, Chen H, Jin M (2010) Identification and characterization of IgA1 protease from Streptococcus suis. Vet Microbiol 140:171-175 
9. Corthesy B (2013) Role of secretory IgA in infection and maintenance of homeostasis. Autoimmun Rev 12:661-665

10. Bek-Thomsen M, Poulsen K, Kilian M (2012) Occurrence and evolution of the paralogous zinc metalloproteases IgA1 protease, ZmpB, ZmpC, and $\mathrm{ZmpD}$ in Streptococcus pneumoniae and related commensal species. MBio 3:e00303-e00312

11. Weinert LA, Chaudhuri RR, Wang J, Peters SE, Corander J, Jombart T, Baig A, Howell KJ, Vehkala M, Valimaki N, Harris D, Chieu TT, Van Vinh Chau N, Campbell J, Schultsz C, Parkhill J, Bentley SD, Langford PR, Rycroft AN, Wren BW, Farrar J, Baker S, Hoa NT, Holden MT, Tucker AW, Maskell DJ, Consortium BRT (2015) Genomic signatures of human and animal disease in the zoonotic pathogen Streptococcus suis. Nat Commun 6:6740

12. Chen Y, Hayashida A, Bennett AE, Hollingshead SK, Park PW (2007) Streptococcus pneumoniae sheds syndecan-1 ectodomains through ZmpC, a metalloproteinase virulence factor. J Biol Chem 282:159-167

13. Govindarajan B, Menon BB, Spurr-Michaud S, Rastogi K, Gilmore MS, Argueso P, Gipson IK (2012) A metalloproteinase secreted by Streptococcus pneumoniae removes membrane mucin MUC16 from the epithelial glycocalyx barrier. PLoS One 7:e32418

14. Oggioni MR, Memmi G, Maggi T, Chiavolini D, lannelli F, Pozzi G (2003) Pneumococcal zinc metalloproteinase $Z \mathrm{mpC}$ cleaves human matrix metalloproteinase 9 and is a virulence factor in experimental pneumonia. Mol Microbiol 49:795-805

15. Surewaard BG, Trzcinski K, Jacobino SR, Hansen IS, Vughs MM, Sanders EA, van der Ende A, van Strijp JA, de Haas CJ (2013) Pneumococcal immune evasion: ZmpC inhibits neutrophil influx. Cell Microbiol 15:1753-1765

16. Leib SL, Leppert D, Clements J, Tauber MG (2000) Matrix metalloproteinases contribute to brain damage in experimental pneumococcal meningitis. Infect Immun 68:615-620

17. Park PW, Foster TJ, Nishi E, Duncan SJ, Klagsbrun M, Chen Y (2004) Activation of syndecan-1 ectodomain shedding by Staphylococcus aureus alpha-toxin and beta-toxin. J Biol Chem 279:251-258

18. Park PW, Pier GB, Preston MJ, Goldberger O, Fitzgerald ML, Bernfield M (2000) Syndecan-1 shedding is enhanced by LasA, a secreted virulence factor of Pseudomonas aeruginosa. J Biol Chem 275:3057-3064

19. Warrens AN, Jones MD, Lechler RI (1997) Splicing by overlap extension by PCR using asymmetric amplification: an improved technique for the generation of hybrid proteins of immunological interest. Gene 186:29-35

20. Takamatsu D, Osaki M, Sekizaki T (2001) Thermosensitive suicide vectors for gene replacement in Streptococcus suis. Plasmid 46:140-148

21. Quan J, Tian J (2009) Circular polymerase extension cloning of complex gene libraries and pathways. PLoS One 4:e6441

22. Burnette WN (1981) "Western blotting": electrophoretic transfer of proteins from sodium dodecyl sulfate-polyacrylamide gels to unmodified nitrocellulose and radiographic detection with antibody and radioiodinated protein A. Anal Biochem 112:195-203

23. Charland N, Harel J, Kobisch M, Lacasse S, Gottschalk M (1998) Streptococcus suis serotype 2 mutants deficient in capsular expression. Microbiology 144:325-332

24. Gottschalk M, Higgins R, Boudreau M (1993) Use of polyvalent coagglutination reagents for serotyping of Streptococcus suis. J Clin Microbiol 31:2192-2194

25. Li Y, Martinez G, Gottschalk M, Lacouture S, Willson P, Dubreuil JD, Jacques M, Harel I (2006) Identification of a surface protein of Streptococcus suis and evaluation of its immunogenic and protective capacity in pigs. Infect Immun 74:305-312

26. Ye C, Zhu X, Jing H, Du H, Segura M, Zheng H, Kan B, Wang L, Bai X, Zhou Y, Cui Z, Zhang S, Jin D, Sun N, Luo X, Zhang J, Gong Z, Wang X, Wang L, Sun H, Li Z, Sun Q, Liu H, Dong B, Ke C, Yuan H, Wang H, Tian K, Wang Y, Gottschalk M, Xu J (2006) Streptococcus suis sequence type 7 outbreak, Sichuan, China. Emerg Infect Dis 12:1203-1208

27. Menon BB, Govindarajan B (2013) Identification of an atypical zinc metalloproteinase, ZmpC, from an epidemic conjunctivitis-causing strain of Streptococcus pneumoniae. Microb Pathog 56:40-46

28. Lalonde M, Segura M, Lacouture S, Gottschalk M (2000) Interactions between Streptococcus suis serotype 2 and different epithelial cell lines. Microbiology 146:1913-1921

29. Meng F, Wu NH, Seitz M, Herrler G, Valentin-Weigand P (2016) Efficient suilysin-mediated invasion and apoptosis in porcine respiratory epithelial cells after streptococcal infection under air-liquid interface conditions. Sci Rep 6:26748
30. Fulcher ML, Gabriel S, Burns KA Yankaskas JR, Randell SH (2005) Welldifferentiated human airway epithelial cell cultures. Methods Mol Med 107:183-206

31. Seitz M, Beineke A, Seele J, Fulde M, Valentin-Weigand P, Baums CG (2012) A novel intranasal mouse model for mucosal colonization by Streptococcus suis serotype 2. J Med Microbiol 61:1311-1318

32. Auger JP, Fittipaldi N, Benoit-Biancamano MO, Segura M, Gottschalk M (2016) Virulence studies of different sequence types and geographical origins of Streptococcus suis serotype 2 in a mouse model of infection. Pathogens 5:E48

33. Silva LM, Baums CG, Rehm T, Wisselink HJ, Goethe R, Valentin-Weigand P (2006) Virulence-associated gene profiling of Streptococcus suis isolates by PCR. Vet Microbiol 115:117-127

34. Pallares FJ, Halbur PG, Schmitt CS, Roth JA, Opriessnig T, Thomas PJ, Kinyon JM, Murphy D, Frank DE, Hoffman LJ (2003) Comparison of experimental models for Streptococcus suis infection of conventional pigs. Can J Vet Res 67:225-228

35. Epperson TK, Patel KD, McEver RP, Cummings RD (2000) Noncovalent association of P-selectin glycoprotein ligand-1 and minimal determinants for binding to P-selectin. J Biol Chem 275:7839-7853

36. Freissler E, Meyer auf der Heyde A, David G, Meyer TF, Dehio C (2000) Syndecan-1 and syndecan-4 can mediate the invasion of OpaHSPGexpressing Neisseria gonorrhoeae into epithelial cells. Cell Microbiol 2:69-82

37. Teng YH, Aquino RS, Park PW (2012) Molecular functions of syndecan-1 in disease. Matrix Biol 31:3-16

38. Zhang A, Mu X, Chen B, Han L, Chen H, Jin M (2011) IgA1 protease contributes to the virulence of Streptococcus suis. Vet Microbiol 148:436-439

39. Cerutti A, Cols M, Gentile M, Cassis L, Barra CM, He B, Puga I, Chen K (2011) Regulation of mucosal IgA responses: lessons from primary immunodeficiencies. Ann NY Acad Sci 1238:132-144

40. Janoff EN, Rubins JB, Fasching C, Charboneau D, Rahkola JT, Plaut AG, Weiser JN (2014) Pneumococcal IgA1 protease subverts specific protection by human IgA1. Mucosal Immunol 7:249-256

41. Pabst O (2012) New concepts in the generation and functions of IgA. Nat Rev Immunol 12:821-832

42. Fu L, Zhao J, Lin L, Zhang Q, Xu Z, Han L, Xie C, Zhou R, Jin M, Zhang A (2016) Characterization of $\operatorname{IgA} 1$ protease as a surface protective antigen of Streptococcus suis serotype 2. Microbes Infect 18:285-289

43. Auger JP, Chuzeville S, Roy D, Mathieu-Denoncourt A, Xu J, Grenier D, Gottschalk M (2017) The bias of experimental design, including strain background, in the determination of critical Streptococcus suis serotype 2 virulence factors. PLoS One 12:e0181920

44. Jobin MC, Fortin J, Willson PJ, Gottschalk M, Grenier D (2005) Acquisition of plasmin activity and induction of arachidonic acid release by Streptococcus suis in contact with human brain microvascular endothelial cells. FEMS Microbiol Lett 252:105-111

45. Tanabe S, Grenier D (2009) Endothelial cell/macrophage cocultures as a model to study Streptococcus suis-induced inflammatory responses. FEMS Immunol Med Microbiol 55:100-106

46. Vandooren J, Van den Steen PE, Opdenakker G (2013) Biochemistry and molecular biology of gelatinase B or matrix metalloproteinase-9 (MMP-9): the next decade. Crit Rev Biochem Mol Biol 48:222-272

47. Yabluchanskiy A, Ma Y, Iyer RP, Hall ME, Lindsey ML (2013) Matrix metalloproteinase-9: many shades of function in cardiovascular disease. Physiology (Bethesda) 28:391-403

48. Carlow DA, Gossens K, Naus S, Veerman KM, Seo W, Ziltener HJ (2009) PSGL-1 function in immunity and steady state homeostasis. Immunol Rev 230:75-96

49. Jonckheere N, Van Seuningen I (2008) The membrane-bound mucins: how large O-glycoproteins play key roles in epithelial cancers and hold promise as biological tools for gene-based and immunotherapies. Crit Rev Oncog 14:177-196

50. Linden SK, Sutton P, Karlsson NG, KorolikV, McGuckin MA (2008) Mucins in the mucosal barrier to infection. Mucosal Immunol 1:183-197

51. Perez BH, Gipson IK (2008) Focus on molecules: human mucin MUC16. Exp Eye Res 87:400-401

52. Bernfield M, Gotte M, Park PW, Reizes O, Fitzgerald ML, Lincecum J, Zako M (1999) Functions of cell surface heparan sulfate proteoglycans. Annu Rev Biochem 68:729-777 
53. Chiavolini D, Memmi G, Maggi T, lannelli F, Pozzi G, Oggioni MR (2003) The three extra-cellular zinc metalloproteinases of Streptococcus pneumoniae have a different impact on virulence in mice. BMC Microbiol 3:14

54. Hayashida A, Amano S, Park PW (2011) Syndecan-1 promotes Staphylococcus aureus corneal infection by counteracting neutrophil-mediated host defense. J Biol Chem 286:3288-3297

55. Yamaguchi M, Nakata M, Sumioka R, Hirose Y, Wada S, Akeda Y, Sumitomo T, Kawabata S (2017) Zinc metalloproteinase ZmpC suppresses experimental pneumococcal meningitis by inhibiting bacterial invasion of central nervous systems. Virulence 8:1516-1524
56. Slater JD, Allen AG, May JP, Bolitho S, Lindsay H, Maskell DJ (2003) Mutagenesis of Streptococcus equi and Streptococcus suis by transposon Tn917. Vet Microbiol 93:197-206

57. Ye $C$, Zheng $H$, Zhang J, Jing $H$, Wang $L$, Xiong $Y$, Wang $W$, Zhou Z, Sun Q, Luo X, Du H, Gottschalk M, Xu J (2009) Clinical, experimental, and genomic differences between intermediately pathogenic, highly pathogenic, and epidemic Streptococcus suis. J Infect Dis 199:97-107

58. Okura M, Osaki M, Fittipaldi N, Gottschalk M, Sekizaki T, Takamatsu D (2011) The minor pilin subunit Sgp2 is necessary for assembly of the pilus encoded by the srtG cluster of Streptococcus suis. J Bacteriol 193:822-831
Ready to submit your research? Choose BMC and benefit from:

- fast, convenient online submission

- thorough peer review by experienced researchers in your field

- rapid publication on acceptance

- support for research data, including large and complex data types

- gold Open Access which fosters wider collaboration and increased citations

- maximum visibility for your research: over 100M website views per year

At BMC, research is always in progress.

Learn more biomedcentral.com/submissions 